\title{
A Bragg Grating Sensor for Detection of Monolayers at a Surface
}

\author{
R. M. Parker ${ }^{1,2}$, J. C. Gates ${ }^{1}$, P. G. R. Smith ${ }^{1}$, M. C. Grossel ${ }^{2}$ \\ ${ }^{1}$ Optoelectronics Research Centre, ${ }^{2}$ School of Chemistry, University of Southampton, Highfield, Southampton, United Kingdom, SO17 1BJ. \\ rmp203@soton.ac.uk
}

Planar waveguides can be written with a UV-laser into photosensitized silica to produce a wide range of optical devices. Careful modulation of two interfering beams allows Bragg gratings to be directly written into the channel (Figure 1.a). These Bragg gratings are inherently sensitive to temperature and strain. However etching away the surface exposes the mode within the grating to its surroundings. The corresponding observed shift in Bragg wavelength can be used to detect changes in this environment [1]. Figure 1.b shows a large wavelength shift due to two analytes of markedly different refractive index. This demonstrates the apodised spectral response that in practice allows resolution of sub-picometer shifts in wavelength.

a)

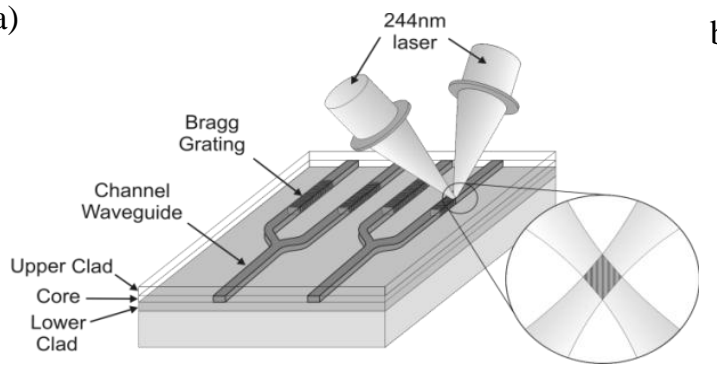

)

$$
\text { ह }
$$

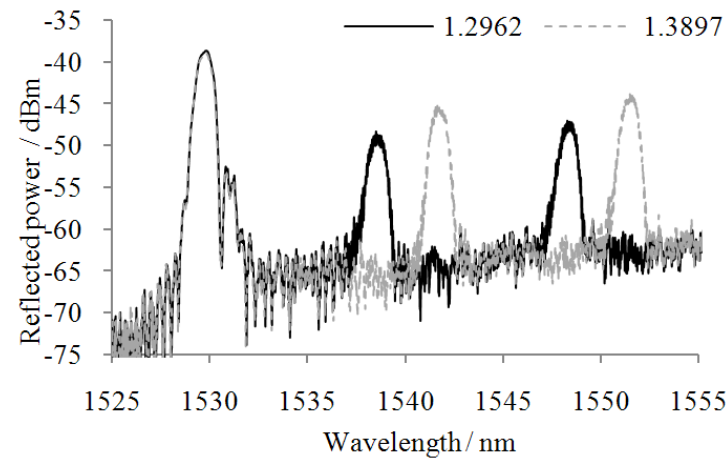

Fig. 1.a) Schematic of direct UV writing of planar waveguides containing Bragg gratings into germanium doped silica.

Fig. 1.b) The spectral response for analytes with indices of 1.2962 (black) and 1.3897 (grey); showing the shift in the pair of Bragg gratings on change in effective index, compared to the temperature reference grating at $\sim 1530 \mathrm{~nm}$.

To enhance detection a thin layer of tantalum pentoxide $(\sim 40 \mathrm{~nm})$ over the etched region of the waveguide pulls up the guided mode, increasing the sensitivity by an order of magnitude. This exploits the much higher refractive index of tantalum pentoxide compared to that of silica [2]. This sensitivity to changes in refractive index was found to be in the order of $10^{-6}$; an order of magnitude more sensitive than the shift expected to be observed on tethering a molecular monolayer onto the surface.

The sensor was incorporated into a microfluidic system as this offered increased reproducibility and control over that of an open system. Switching between solvent analytes (e.g. acetone, isopropanol, methanol, water) within the microfluidic channel resulted in a series of well resolved changes in the effective index of the grating. However, on closer examination it was found that the previous analyte impacted the following measurement. This is attributed to the formation and displacement of monolayers on the surface that demonstrate slower kinetics than the bulk fluid.

The exposed waveguide surface can be readily functionalised via an alkylsilane linker to form an organic monolayer on the surface; allowing a wide range of functional moieties to be introduced. This allows for the design of specific interactions to occur at the surface that would enhance the chemical specificity of the system.

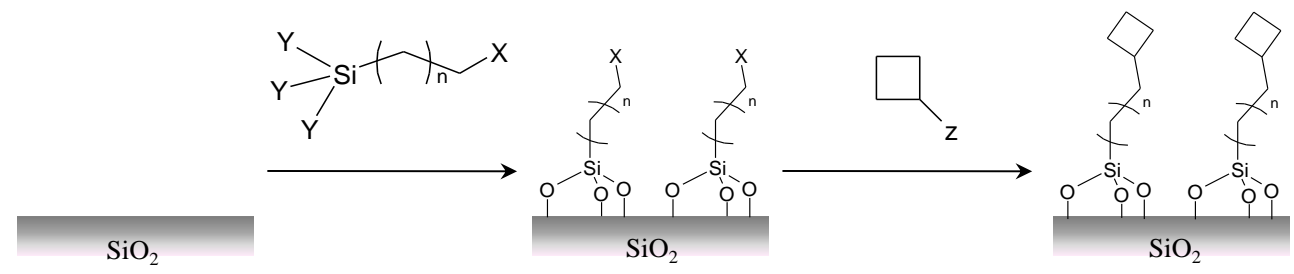

Fig. 2 A generic reaction scheme for the synthesis of organic surfaces on silica, using an alkylsilane linker.

The device is a highly sensitive refractive index sensor with the potential to detect down to monolayer resolutions and is able to see subtle chemical surface effects. We shall present the latest developments to introduce chemical specificity through functional self assembled monolayer (SAM) surfaces and exploration of the chemical interactions at these surfaces.

\section{References}

[1] I.J.G. Sparrow, G. D. Emmerson, C.B.E. Gawith, P.G.R. Smith, M. Kaczmarek, A. Dyadyusha, "First order phase change detection using planar waveguide Bragg grating refractometer", Appl. Phys. B, 81, 1-4, (2005).

[2] G.R. Quigley, R.D. Harris, J.S. Wilkinson, "Sensitivity enhancement of integrated optical sensors by use of thin high-index films", Applied Optics, 38, 6036-6039, (1999). 